Research Article

\title{
Sports Event Level Measurement Indicator System Using Multisensor Information Fusion
}

\author{
Weiwei $Y u^{1,2}$ and Jinming Xing $\mathbb{D}^{3}$ \\ ${ }^{1}$ School of Economics and Management, Dalian University of Technology, Dalian 116024, China \\ ${ }^{2}$ Suan Sunan Rajabhat University, Bangkok 10300, Thailand \\ ${ }^{3}$ School of Physical Education, Northeast Normal University, Changchun 130024, China
}

Correspondence should be addressed to Jinming Xing; xingjm100@nenu.edu.cn

Received 11 August 2021; Revised 21 September 2021; Accepted 13 October 2021; Published 1 November 2021

Academic Editor: Mu Zhou

Copyright (c) 2021 Weiwei Yu and Jinming Xing. This is an open access article distributed under the Creative Commons Attribution License, which permits unrestricted use, distribution, and reproduction in any medium, provided the original work is properly cited.

In view of the imperfection of the measurement index of the level of sports events, the coverage rate of the measurement index is low and the stability is poor. Therefore, this paper puts forward a sports event level measurement index system based on multisensor information fusion. First, the simulated annealing algorithm is used to cluster the grouped sensors and fuse the optimal Bayesian estimation of compatible sensors. Second, the relative entropy measure method is used to expand the compatibility measure of the sensor information in the group, and the optimal Bayesian estimation value of the consistent measure test is obtained. The outliers are eliminated, the optimal fusion value is obtained by the overall weighted statistical fusion, and the alternative measure index system is constructed. Finally, analytic hierarchy process (AHP) is used to calculate the weight of each alternative index, so as to achieve the final measurement index. The results show that the standard deviation of clustering average energy consumption is low, and the energy loss is small. The system can effectively construct the measurement index, and the index coverage rate is as high as $95 \%$.

\section{Introduction}

Sporting Event is a hotspot in physical education and sports policy researches. Sporting Event promotes the development of physical education [1]. As a major part of the school work, it represents the progress of a school's physical education [2]. Development of Sporting Event Promoting School National Fitness Movement can improve students' understanding and quantity of sports skills. Also, it helps to create an excellent product of the integrity of the collective honor. Therefore, it has good teaching significance. At present, the Sporting Event level measurement indicators have a small scope of application, and there is a certain defect in terms of indicator coverage. Sporting Event itself has a strong linkage effect [3]. Therefore, Sporting Event can not only bring a lot of people and promote direct consumption but also lead the development of sports industry, thus, promoting the development of other industries. On this basis, the collaborative development of regional Sporting Event is bound to lead the development of regional sports industry and the development of regional economy.

The establishment of any index system must first have a basic framework on which specific indicators depend. This basic framework is actually an interpretation system corresponding to specific objects, also known as theoretical framework or theoretical model. According to the existing research results, the general evaluation index system models include "structure function" model, "input-output" model, and "condition result" model. The basic framework is the soul of the index system. Without the base wood framework, it cannot be called "system." The design of the basic theoretical framework of the index system is the most critical link in the construction of the index system. For sports modernization, the sports modernization index system should not only reflect the comprehensiveness of the sports system structure but also reflect the basic characteristics of modern sports development and the three basic cognitive representations of modernization. Therefore, it is difficult to reflect the face 
of sports modernization according to a specific model. It can be seen that the synergy of Beijing-Tianjin-Hebei Sporting Event has an important impact on the development of the Beijing-Tianjin-Hebei Sports industry and the development of Beijing-Tianjin. Most of the risk management research in various fields has explored the risk indicator system varying degrees. However, there are very few literatures built in detail the risk identification and risk indicator system for two work sections. Obviously, the result of this is more conducive to the study of the context, which facilitates follow-up theoretical analysis. The risk factors that trust problems in Sporting Event will be initially identified by real-world research, consulting related experts, checking literature, and historical information. Then, according to the Sporting Event, trust risk is from the event participation in the main body itself, the event system, the event system, the overall operation environment, etc.; this paper is initially recognized in the trust risk of Sporting Event. Then, according to the trust risk of sports events, this paper preliminarily identifies the trust risk of sports events from the aspects of the event participants themselves, the event system, the event system, and the overall operation environment of the event.

In this regard, this paper proposes a study of Sporting Event level measurement indicators based on multisensor information fusion. Its innovation is to calculate the weight of each alternative indicator by hierarchical analysis and determine the final measurement index. The best fusion output value is obtained using the Bayesian estimation method. The multisensor fusion method is used to fuse the multiangle information. Compared with the information collected by the single sensor, the Sporting Event level measurement index system based on multisensor information fuses is studied, and more comprehensive measurement indicators are constructed.

(1) This paper proposes a research on the Sporting Event level measurement indicator system based on multisensor information fusion. The optimal Bayesian methods estimate with compatibility sensors is fused by simulating an annex algorithm clustering group sensor

(2) The weight of each alternative indicator is calculated by hierarchical analysis, and the final measurement indicator is determined. By calculating the score of the optional indicator, the range of values is obtained

(3) The priority distribution is obtained by utilizing great likelihood estimation, and the best fusion output value is obtained by using the Bayesian estimation method. Finally, the experimental results of different data sets show the effectiveness of the methods of this paper

\section{Related Work}

Literature [4] proposes a large span spatial structure stress recognition method based on multitype sensor information fusion. It is proposed to solve the acquisition method of large span spatial structural stress distribution and reduce the number of strain sensors in the strain sensor by using the measured information and information fusion methods of multiple types of sensors. But the method is not so stable. Literature [5] method proposes a new DEMS method to solve data loss problems in the mobile sensor network. DEMS method uses a virtual static sensor to solve the problem of sensitive sensor packets. At the same time, the mobile sensor network is also solved, and when the sensor position changes, the problem is lacking or loses history. However, the method has incomplete index. Literature [6] proposed a hierarchical weight decision analysis method. In the case where the indicator is initially determined, a hierarchical analysis method is used to assign the weight of each indicator. Finally, through the method of seeking a geometric average, the relative weights of each indicator in the modernization index system of the capital are obtained. However, the energy loss of this method is large. The literature [7] method proposes the theoretical model of public service of large sports venues and obtains the required data of the verification model through the questionnaire survey method. In addition, the structural equation model is used to verify the previously proposed influencing factors. Finally, based on the influencing factors, the propulsion strategy of the public service of large sports venues is proposed, but the prediction accuracy of this method is low. Literature [8] proposes a method of solving a sequential parameter based on gray correlation degree and attribute. The gray correlation between the state parameters and decision variables is used as a reference and basis for judging state parameters. Through the calculation of gray correlation, the preliminary screening of state parameters can be performed, the state parameters with high correlation with the decision parameters are sequentially parameters, and the professional sports club value chain system synergies are further evaluated in sequence parameters. However, the horizontal measurement error of this method is large.

Therefore, this paper proposes a research on sports event level measurement index system based on multisensor information fusion. The weight of each alternative index is calculated by analytic hierarchy process to determine the final measurement index. The risk function is obtained by constructing the risk function. Through the largest consistent sensor group, the largest associated group sensor is obtained, the total fusion group information is combed, and the total fusion output is obtained.

\section{Multisensor Information Fusion Method}

The Sporting Event Horizontal Measurement indicator System Based on Multisensor Information Fusion mainly includes information fusion. This paper establishes measurable indicator system and identifies metric weights and determinations [9]. Further, the Sporting Event information collected by the sensor is fused by multisensor information fusion method. The Sporting Event Horizontal Preparation Surveying Indicator System is constructed according to this information; the weight of each alternative indicator is calculated by hierarchical analysis, and the final measurement indicator is determined. 
3.1. Multisensor Clustering Group Based on Simulation Annealing Algorithm. Simulation Annealing Algorithm has the advantages of failing with the overall optimal solution relative to other intelligent algorithms in solving problems and can find global optimal solutions. Therefore, this article details the mathematical model of Simulation Annealing Algorithm and its optimistic method [10]. This proves Simulation Annealing Algorithm to find the global optimal solution. Further, this paper uses analog degenerative algorithm to deploy a polymerization grouping group, and the specific steps are as follows:

Step 1. Select the lead node.

Step 2. Start the external cycle, broadcast the Hello message in the sensor network. An additional cluster is selected according to the node other than the receiving signal, and each node can only select one cluster. Set the initial cluster as $H_{0}\left(H_{1}, H_{2}, \cdots, H_{N}\right)$. When it is an initial solution, set the initial temperature $T_{0}$, and the iteration times $k$ of each $T$.

Step 3. Start the internal cycle, order the current granary $\alpha$ $H_{\alpha}=H_{0}$, the current temperature $T_{\alpha}=T_{0}$.

Step 4. Select several nodes to readd clustering, formation of $\beta H_{\beta}$. Get the solution of $H_{\alpha}$ and $H_{\beta}$, and then get $\Delta h=$ $h\left(H_{\beta}\right)-h\left(H_{\alpha}\right)$. If $h \leq 0$, then $H_{\alpha}=H_{\beta}$. Conversely, in accordance with Monte Carlo accept criteria storage $H_{\alpha}$. According to this guideline, the transfer probability definitions $H_{\alpha}$ and $H_{\beta}$ are

$$
Q\left(H_{\alpha} \longrightarrow H_{\beta}\right)=\Delta h \leq 0,
$$

where $\Delta h$ is the measure function of sports event level.

Step 5. When the number of iterations is greater than or equal to $t$, terminate the inner loop [11-13]. When $T_{\alpha}$ decreases, it is necessary to determine whether the external circulation is terminated. If not, return to step 3 and continue the internal circulation.

Step 6. If the outer loop condition is met, terminate the algorithm and obtain the approximate optimal clustering group $H_{\alpha}$.

3.2. Multisensor Information Fusion Based on Optimal Bayesian Estimation. After clustering and grouping, the relative entropy measurement method is used to expand the compatibility measure of the sensor information in the group to accurately present the compatibility between sensors [14, 15]. All information needs to be standardized between the compatibility measures. Assuming that the number of sensors in a group is $m$, if the length of each sensor data sequence is $n$, the information processing formula is as follows:

$$
y_{i j}^{\prime}=\left|\frac{y_{i j}-\bar{y}_{i}}{\delta_{i}}\right|, i=1 \text {, }
$$

where expectation is $\bar{y}_{i}$; the variance is $\delta_{i}$; the original information is $y_{i j}$. Standardized information is $y_{i j}^{\prime}$. After arranging $y_{i j}^{\prime}$ from large to small, the compatibility between sensors is measured by relative entropy measure, so that the relative information of $i$ and $j$ is as follows:

$$
E_{i j}=\left|\sum_{k=1}^{n}\left[1-\frac{y_{i j}}{\sum_{k=1}^{n} \bar{y}_{i}}\right]\right|=1,2, \cdots, m,
$$

where $E_{i j}$ represents the data fusion coefficient. $m$ represents the network node of the sensor, $E_{i j} \neq E_{j i}$. $E_{j i}$ is the relative compatibility between sensors. The formula for accurately depicting the compatibility of the sensor is as follows:

$$
\rho_{i j}=\frac{\min \left(E_{i j}, E_{j i}\right)}{\max \left(E_{i j}, E_{j i}\right)}, i, j=1,2, \cdots, m,
$$

where in the case of $\rho_{i j}=0$, it means that $i$ and $j$ are incompatible, that is, there is a contradiction between information and information fusion cannot be carried out.

When $\rho_{i j}=1$, it means that $i$ and $j$ are compatible, that is, there is redundant information and information fusion can be carried out.

In the case of $0<\rho_{i j}<1$, it represents that $i$ and $j$ are partially compatible, that is, the information is complementary, and it needs to be analyzed to judge whether they can be fused [16].

The compatibility measure matrix is $R_{c}=\left\lfloor c_{i j}\right\rfloor$ and the compatibility degree is $c_{i j}$.

After $\varepsilon_{c}\left(0<\varepsilon_{c}<1\right)$ determines $c_{i j}$, follow the Zodiac Normal Statistical Strategy, shaping the consistent measurement operator as follows

$$
c_{i j}=\left\{\begin{array}{l}
1, \text { if } \rho_{i j} \geq \varepsilon_{c}, \\
0, \text { if } \rho_{i j}<\varepsilon_{c} .
\end{array}\right.
$$

where $c_{i j}$ indicates compatibility $\varepsilon_{c}$ represents the information fusion output value of the sensor. After completing the compatibility measure, the prior distribution is obtained by maximum likelihood estimation, and then the best fusion output value is obtained by the Bayesian estimation method [17]. The sensor model is represented by Gaussian distribution. Assuming that the number of sensors is $L$, the model of sensor $i$ is

$$
P_{i}(x)=c_{i j}\left(-\frac{1}{2}\left(\frac{y-\bar{y}_{i}}{\delta_{i}}\right)\right), i=1,2, \cdots, j,
$$

where $P_{i}(x)$ represents the characteristic quantity $\delta_{i}$ indicates system suitability. Use the model of formula (6) to fuse information. The formula is as follows: 
$p\left(\theta \mid y_{1}, y_{2}, \cdots, y_{L}\right)=\prod_{i=1}^{N_{L}}\left[p\left(\theta \mid y_{i}\right)\right]=\frac{\prod_{i=1}^{N_{L}}\left[p\left(y_{i} \mid \theta\right) \cdot p(\theta)\right]}{\int p\left(\left|y_{1}, y_{2}, \cdots, y_{L}\right| \theta\right) \cdot p(\theta)}$,

where the optimal fusion output is $\theta$; according to Bayesian estimation theory, the optimal estimation value is $\widehat{\theta}=\int_{\Theta} \theta$ - $p\left(\theta \mid y_{1}, y_{2}, \cdots, y_{L}\right)$. The value parameter space of $\theta$ is $\Theta$. According to the theory of maximum likelihood estimation, we know the distribution of $\theta$ is $N\left(u_{0}, \delta_{0}^{2}\right)$.

$$
\begin{aligned}
& u_{0}=\frac{\sum_{i=1}^{L}\left(1 / \delta_{i}^{2} \cdot \bar{y}_{i}\right)}{\sum_{i=1}^{L}\left(1 / \delta_{i}^{2}\right)} . \\
& \delta_{0}^{2}=\frac{1}{N} \sum_{i=1}^{L}\left[\frac{1}{\delta_{i}^{2}}\right] .
\end{aligned}
$$
follows:

Build a risk function to get $\widehat{\theta}$, and the risk function is as

$$
\lambda(\theta, \widehat{\theta})=(\theta-\theta \wedge)^{2}=\left(\theta-\int_{\Theta} \theta \cdot p\left(\theta \mid y_{1}, y_{2}, \cdots, y_{L}\right)\right)^{2} .
$$

Let $\partial e(\theta) /\left.\partial \theta\right|_{\theta=\widehat{\theta}}=0$. To calculate $\widehat{\theta}$, the formula is as follows:

$$
\widehat{\theta}=\frac{b+\sqrt{b^{2}+4 a}}{2 a}
$$

where $b$ is parameter model of cluster sensor, and $a$ is the combination parameters of cluster sensors. This method is used to process the $N$ group sensor to obtain the best Bayesian estimation of each group of sensors $\widehat{y}_{i}, i=1,2, \cdots, N$ [18].

3.3. Multisensor Distribution Integration Based on Uniform Measurement. After the clustering processing sensor, each set of sensor information has dispersion. Before the information is integrated, it is necessary to conduct a consistency measurement, and the fusion output value of the $\mathrm{N}$ group sensor is $\widehat{y}_{i}$, the variance is $\widehat{\delta}_{i}^{2}$. Follow the Zodiac Normal Statistical Strategy, shaping the consistent measurement operator as follows:

$$
d_{i j}\left\{\begin{array}{l}
1-\frac{\left|\widehat{y}_{i}-\widehat{y}_{j}\right|}{3 \widehat{\delta}_{i}}, \text { if } \widehat{y}_{i} \neq \widehat{y}_{j}, \text { and }\left|\widehat{y}_{i}-\widehat{y}_{j}\right|<3 \widehat{\delta}_{i}, \\
1-\frac{\left|\widehat{\delta}_{i}-\widehat{\delta}_{j}\right|}{3 \widehat{\delta}_{i}}, \text { if } \widehat{y}_{i}=\widehat{y}_{j}, \quad \text { and }\left|\widehat{y}_{i}-\widehat{y}_{j}\right|<3 \widehat{\delta}_{i}, \\
0, \text { else. }
\end{array}\right.
$$

Depict the support of $i$ to $j$ via $d_{i j} . d_{i j}=1$ means that the measured value of $i$ and $j$ is basically the same, and $i$ strongly supports $j[11,19] ; d_{i j} \neq 0$ means the support of $i$ to $j$ is weak. At the same time, the variance is inversely proportional [20]; $d_{i j}=0$ means $i$ and $j$ have no consistent measurement. According to $d_{i j}$, calculate the corresponding uniform association array $R_{r}=\left[\tau_{i j}\right]$, and the consistent correlation is $\tau_{i j}$.

$$
\tau_{i j}=\left\{\begin{array}{l}
1, \text { if } d_{i j} \geq \varepsilon_{c} . \\
0, \text { if } d_{i j}<\varepsilon_{c} .
\end{array}\right.
$$

where $d_{i j}$ represents characteristic parameter. The maximum consistent sensor group is obtained by $R_{r}$, which makes the maximum number of associated group sensors are $l(0<l<$ $N)$. Integrate this group of information $[21,22]$, get the total fusion output $\widehat{X}$.

The integration steps are as follows:

Step 1. Cluster sensor, get $N$ group sensor. $\bar{s}=\left\{\bar{s}_{i} \mid i=1,2\right.$, $\cdots, N ; 1 \leq N \leq M\}$.

Step 2. Compatibility measurement test $N$ group sensor, go to the wild point $[23,24]$, combine the optimal Bayesian estimate $\widehat{y}_{i}$.

Step 3. The overall consistent measurement test of $\widehat{y}_{i}$ will continue to go to the wild point, and the overall weighted statistical fusion acquisition is the optimal fusion value $\hat{y}$.

Step 4. Get an optional measurement indicator system via $\widehat{y}$.

3.4. Determine the Measurement Indicators. The hierarchical analysis is used to calculate the weight of the measurement indicator. Determine the final measurement indicator system according to the size of the score $[25,26]$. Shaping judgment matrix for subsequent indicator of the same superior indicators $q_{u v}$, make the number of options $\varsigma$, normalize items in $q_{u v}$

$$
\underline{q}_{u v}=\frac{q_{u v}}{\sum_{1}^{\varsigma} q_{u v}}, u, v=1,2, \cdots, a
$$

To process $\underline{q}_{u v}$ in a row-addition manner, the formula is as follows:

$$
\underline{W}_{u}=\sum_{1}^{\varsigma} \underline{q}_{u v}
$$

Normalization vector $\underline{W}=\left(\underline{W}_{1}, \underline{W}_{2}, \cdots, \underline{W}_{\varsigma}\right)^{t}$, get the weight of the lower-level indicator of the lower-level indicator:

$$
W_{u}=\frac{W_{u}}{\sum_{1}^{c} \underline{W}_{v}} .
$$

$W_{v}$ is the basis. By calculating the score of the optional indicator, the score of the score is $[0,1]$, according to the score of alternative indicators [27-29], select the first 15 score to be 
high as the final measurement indicator. The specific structure is shown in Figure 1.

\section{Experimental Analysis and Results}

4.1. Experimental Environment and Data Set. This experiment uses two disclosed data sets for testing, sparse marking data set (1FPs) represented by AVA. Due to the sparse labeling, there is no clear action boundary, and the existing methods are more like an initiation level action identification, weakened timing positioning; at the same time, the operating category is daily atomic action, slow motion, small shape, low tracking difficulty, classification does not require complex people, and the model and environment modeling and reasoning. Densely labeled data set (25FPS) is represented by JHMDB. This type of data set has only one action for each video. Most of the video is that single people are doing some semantic simple repetitive actions, and the action category is related to the background height. The data set used in this paper contains 150 sequences with a resolution of $720 \times 480$. This data set represents the natural action pool in various scenes and viewpoints. By publishing data sets, we hope to encourage further research on this kind of sports action recognition in an unconstrained environment. It is shown in Table 1.

\subsection{Experimental Indicators}

(1) Clustering Average Load Criteria. The higher the standard, the higher the stability, based on the clustering average load criteria under several different methods, the stronger the stability

(2) Energy Loss. According to the comparison of the relationship between the monitoring area and the number of rounds (the first node death), the lowest number of times is small

$$
p=W_{u} \leq\left(x_{L}^{i}-x_{S}^{i}\right)
$$

where $x_{L}^{i}$ represents the detection area, and $x_{S}^{i}$ represents the number of rounds.

(3) Measurement Indicator Coverage. According to the measurement indicator coverage rate under several different methods, the higher the coverage, the more accurate the prediction accuracy

(4) Measurement Indicator Error Rate. According to the measurement indicator error rate under several different methods, the lower the error rate, the lower prediction effect is stronger

$$
\bar{x}=p-\left(X_{n}, Y_{n-\tau}\right),
$$

where $X_{n}$ is the coupling coefficient of the measurement error index. $Y_{n-\tau}$ is the sequence function of measuring error index.

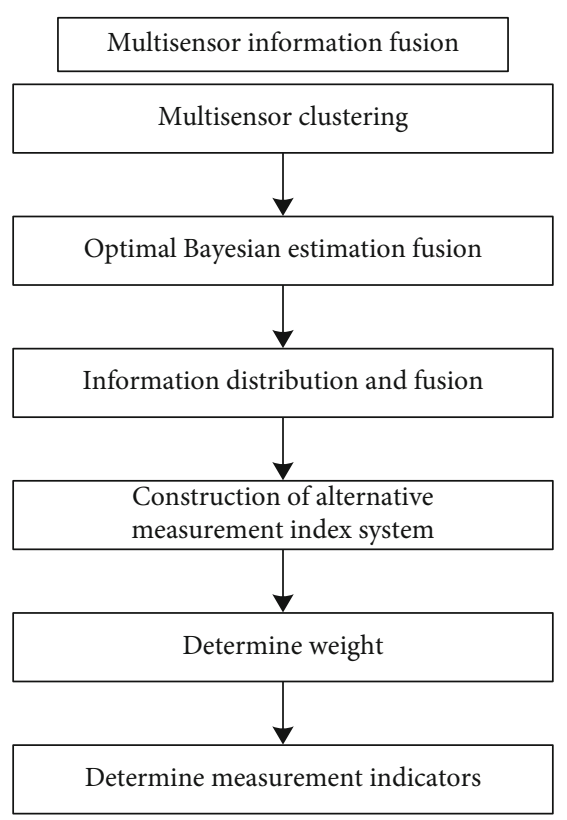

FIgURE 1: Structure diagram of sports event level measurement index system.

(5) Data Transmission Security. According to data transmission security under several different methods, the higher the security, the better the practicality

4.3. Results and Discussion. Taking a body school as an experimental object, the system of this paper is established to establish a measure indicator for the school Sporting Event level and verify the effectiveness of the system.

To test the relationship between the network extension and cluster average energy consumption standard difference, this paper tests the clustering mean load standard deviation (CMLSD) of six systems in different nodes. It is shown in Figure 2.

According to Figure 2, the number of nodes is increasing, that is, the size of the network increases, the larger the network size, and the six-series CMLSD presents an upward trend. The degree of CMLSD growth in this paper is not obvious, the fluctuation is small, the remaining five systems have a large growth, the fluctuations are large, and the stability is poor. Experiments have shown that the CMLSD is minimal and stable in the cluster of the system herein.

This paper tests the number of wheels that appear in different network monitoring area and checks the performance of 6 systems in the extension of network lifecycle. It is shown in Figure 3.

According to Figure 3, as the monitoring area increases, the number of rounds of the first node of six systems has gradually declined. The number of rounds of the first node death in this paper is significantly lower than the remaining five systems. When the monitoring area reaches $400 \mathrm{~m}^{2}$, the number of wheels in the first node in six systems tends to stabilize, and the number of rounds after the system is significantly lower than the remaining five systems. Experiments prove that the number of rounds of the first node death in 
TABLE 1: Related parameter distribution.

\begin{tabular}{|c|c|c|c|c|}
\hline Variable & Mean value & Standard value & Minimum value & Average value \\
\hline Physical quality & 0.135 & 0.167 & 0.633 & 3.644 \\
\hline Behavior feature & 0.358 & 0.546 & 0.477 & 2.588 \\
\hline Spatial planning & 0.535 & 0.366 & 0.435 & 2.577 \\
\hline Joint statistical analysis value & 0.267 & 0.454 & 0.155 & 4.655 \\
\hline Regression analysis value & 0.534 & 0.356 & 0.143 & 3.577 \\
\hline
\end{tabular}

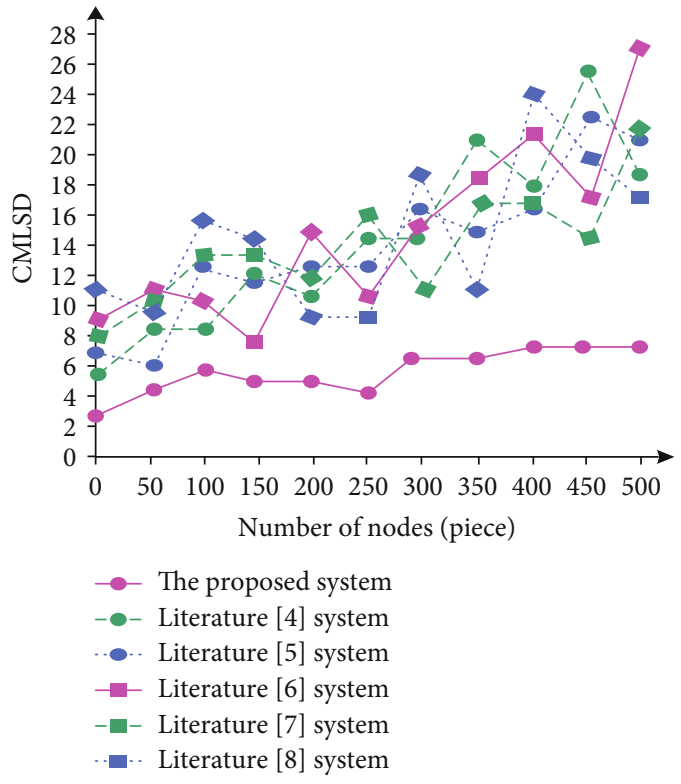

Figure 2: Standard deviation of average load of network nodes and clusters.

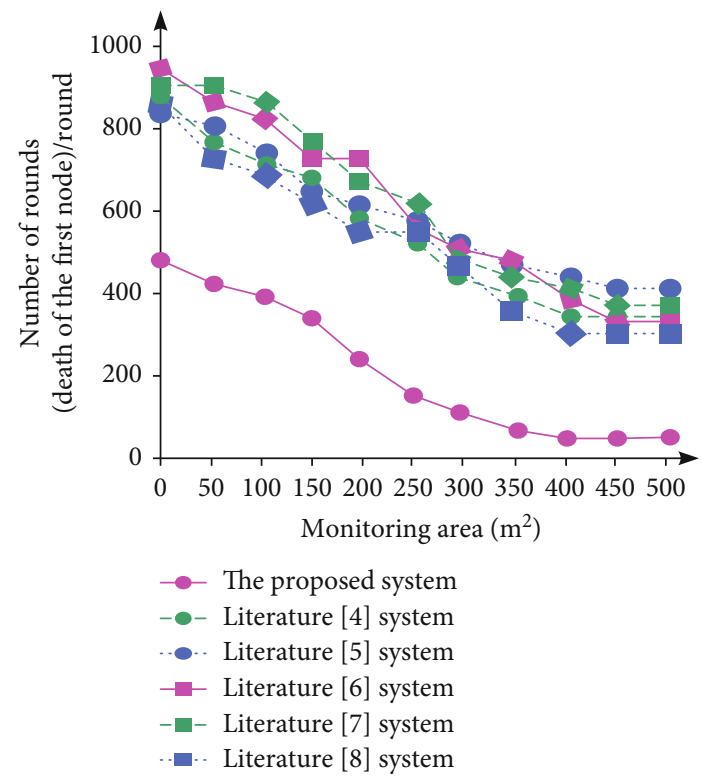

Figure 3: Relationship between monitoring area and number of rounds. this paper has a minimum, indicating that the energy loss in the cluster is small.

After completing the information cluster, the system of this paper gets the Sporting Event level preparation selection indicator. To continue to use this article to calculate the weight of each preparation measurement indicator and calculate the corresponding indicator score, the first 15 indicators in the order of the score are selected as the final measure indicator, alternative metric weights, and score calculation results. It is shown in Table 2 .

According to Table 2, the system in this paper can effectively calculate the weight of the optional measurement indicator and solve the score of each indicator. According to the score from large to small sort score, select the first 15 indicators as the final measurement indicator, in turn is the event insurance, sports slogan and logo, fair competition, funding budget, athlete sports spirit, result statistics and announcements, athletes medical examination, safety and security work, athlete code, staff satisfaction, sports equipment standardization, site facilities, event assessment, event management system, and medical security. Experiments prove that the system of this paper can effectively construct the Sporting Event level measurement indicator.

This paper uses the literature [4] to the literature [8] system to build a Sporting Event horizontal measurement indicator for the school and test the coverage of the measurement indicators of six systems, as shown in Figure 4.

According to Figure 4, the index coverage of literature [4] method is $38 \%$, the index coverage of literature [5] method is $59 \%$, the index coverage of literature [6] method is $46 \%$, the index coverage of literature [7] method is $66 \%$, the index coverage of literature [8] method is $49 \%$, and the index coverage of this method is as high as $97 \%$. It can be seen from this that the coverage rate coverage of this system constructed is significantly higher than the remaining five systems. This shows that the indicator of this article covers a broader field, better service, and predictions such as evaluation and prediction. Excellent measure indicators are applied to assessment and forecasting, etc., which can effectively improve the accuracy of the assessment and forecasting of these methods and lay a solid foundation for the promotion of the Sporting Event level.

According to Figure 5, the index error rate of literature [4] method is $9 \%$, the index error rate of literature [5] method is $9.9 \%$, the index error rate of literature [6] method is $11 \%$, the index error rate of literature [7] method is $13 \%$, the index error rate of literature [8] method is $13.9 \%$, and the index error rate of this method is $4 \%$. It can be seen from this that compared with the systems in other five literature, 
TABLE 2: Calculation results of weights and scores of alternative measurement indicators.

\begin{tabular}{|c|c|c|c|}
\hline Primary indicators (weight) & Secondary indicators (weight) & Tertiary indicators (weight) & Score \\
\hline \multirow{6}{*}{ Basic conditions of the event $(0.43)$} & \multirow{2}{*}{ Event funds $(0.63)$} & Budget $(0.51)$ & 0.91 \\
\hline & & Source of funds (0.49) & 0.65 \\
\hline & \multirow{2}{*}{ Venue facilities (0.55) } & Standardization degree of sports equipment (0.44) & 0.84 \\
\hline & & Safety of site facilities $(0.56)$ & 0.84 \\
\hline & \multirow{2}{*}{ Logistic service $(0.45)$} & Medical security $(0.53)$ & 0.81 \\
\hline & & Security work $(0.47)$ & 0.87 \\
\hline \multirow{14}{*}{$\begin{array}{l}\text { Event organization and management } \\
(0.32)\end{array}$} & \multirow{5}{*}{$\begin{array}{l}\text { Precompetition preparation stage } \\
(0.34)\end{array}$} & $\begin{array}{c}\text { Composition of organization and operation } \\
\text { organization }(0.21)\end{array}$ & 0.80 \\
\hline & & Set event scheme $(0.24)$ & 0.80 \\
\hline & & Event insurance (0.19) & 1.00 \\
\hline & & Site layout (0.19) & 0.80 \\
\hline & & Propaganda work $(0.17)$ & 0.77 \\
\hline & \multirow{4}{*}{ Event stage $(0.32)$} & Coach, team leader, and referee meeting (0.26) & 0.17 \\
\hline & & Physical examination of athletes $(0.25)$ & 0.87 \\
\hline & & Performance statistics and announcement $(0.23)$ & 0.89 \\
\hline & & Risk prevention $(0.26)$ & 0.77 \\
\hline & \multirow{3}{*}{ Event closing stage (0.19) } & Event evaluation $(0.51)$ & 0.83 \\
\hline & & Event file storage (0.49) & 0.75 \\
\hline & & Number of participants $(0.33)$ & 0.78 \\
\hline & Competition level (0.15) & Record breaking situation $(0.31)$ & 0.23 \\
\hline & & Participant satisfaction $(0.36)$ & 0.84 \\
\hline \multirow{8}{*}{ Sports culture $(0.25)$} & \multirow{3}{*}{ Sports system culture $(0.47)$} & Event management system $(0.35)$ & 0.81 \\
\hline & & Traditional sports events $(0.26)$ & 0.67 \\
\hline & & Athlete code $(0.25)$ & 0.86 \\
\hline & \multirow{5}{*}{ Sports spiritual culture $(0.53)$} & Sports slogan and logo $(0.15)$ & 1.00 \\
\hline & & Concept of sports events $(0.17)$ & 0.80 \\
\hline & & Sports ethics $(0.21)$ & 0.75 \\
\hline & & Sportsmanship of athletes $(0.23)$ & 0.90 \\
\hline & & Spirit of fair competition $(0.24)$ & 0.92 \\
\hline
\end{tabular}

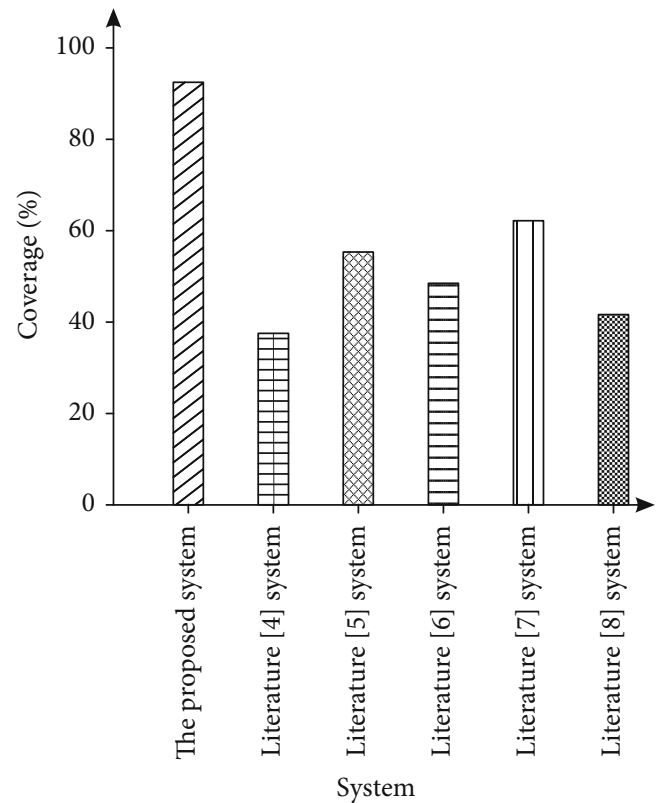

FIGURE 4: Indicators correlation test results.

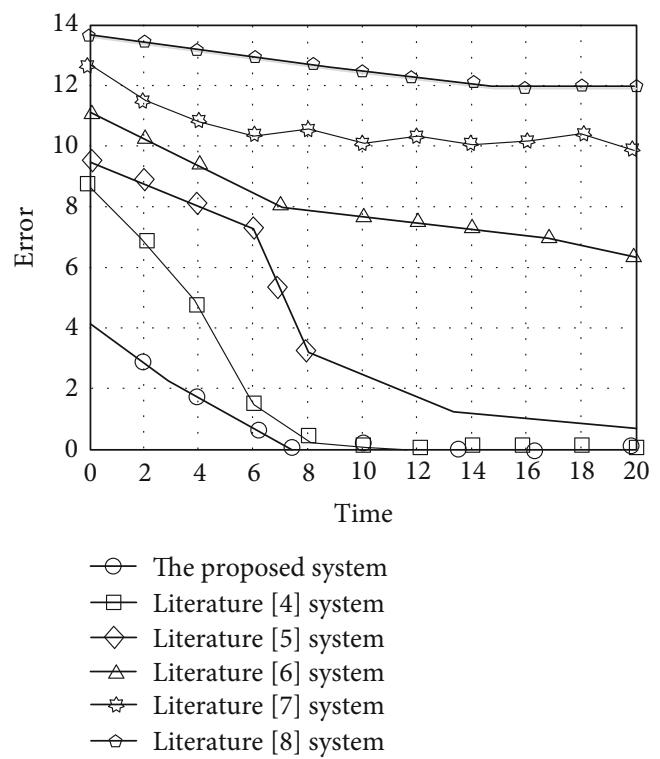

FIgURE 5: Comparison of measurement index errors. 


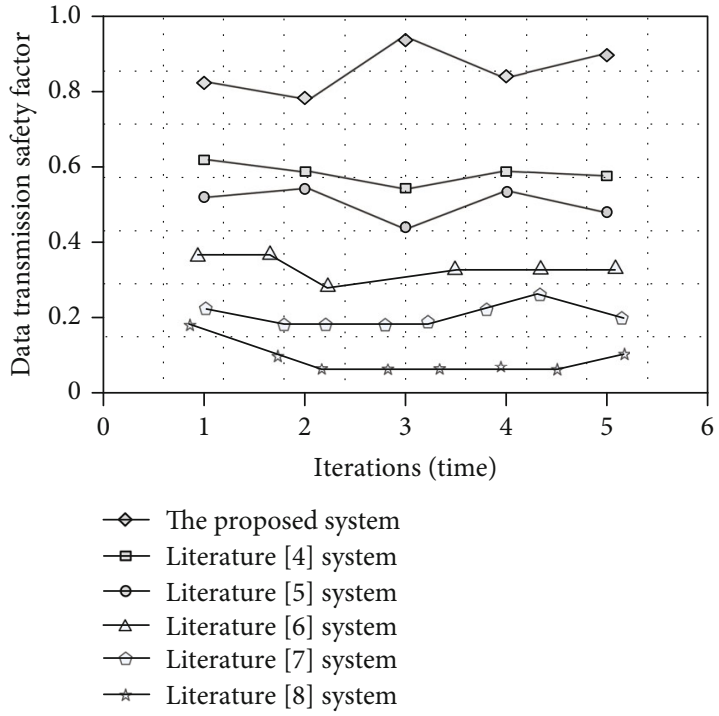

Figure 6: Safety factor of data transmission.

and the Sporting Event measurement indicator error of this paper is lower, indicating that the evaluation prediction of this paper is better and more practical.

According to Figure 6, the transmission safety factor of the method in literature [4] is $0.65 \%$, the transmission safety factor of the method in literature [5] is $0.52 \%$, the transmission safety factor of the method in literature [6] is $0.38 \%$, the transmission safety factor of the method in literature [7] is $0.23 \%$, the transmission safety factor of the method in literature [8] is $0.2 \%$, and the transmission safety factor of the method in this paper is as high as $0.83 \%$. Compared with several other systems, the one in this paper is significantly higher than other systems. It can be seen that the higher the index of using this system to measure the level of sports events, the better the effect of use.

\section{Conclusions}

The imperfection of the Sporting Event level measurement indicator causes problems such as low-conditioned coverage and poor stability. This paper obtains a priori distribution through maximum likelihood estimation and then uses Bayesian estimation to obtain an optimal fusion output value. In addition, this paper uses a hierarchical analysis to calculate the weight of the measurement indicator. Sports policies have been continuously improved, and all regions have significantly improved the organization of sporting events. To enhance the Sporting Event level, this paper studies the Sporting Event level measurement indicator system based on multisensor fusion and reasonably constructs measurement indicators. This can improve the coverage of the indicator and better serve the evaluation and forecasting methods, providing scientific basis for improving the Sporting Event level.

\section{Data Availability}

The data used to support the findings of this study are included within the article. Readers can access the data sup- porting the conclusions of the study from UCF101-24 and JHMDB data set.

\section{Conflicts of Interest}

The authors declare that there is no conflict of interest with any financial organizations regarding the material reported in this manuscript.

\section{Acknowledgments}

This work was supported by National Social Science Fund (20FTYB002), Key projects of Jilin Social Science Fund (2020A10), and Social science research project of Jilin Provincial Department of Education (JJKH20211320SK).

\section{References}

[1] H. Liang, Y. Yandong, and H. Xue, "Sports event risk early warning model based on BP neural network," Statistics and decision making, vol. 34, no. 16, pp. 85-88, 2018.

[2] L. Lu, L. Gang, and H. Haiyan, "Construction and demonstration of evaluation index system for the development of global urban sports industry," Journal of Shanghai Institute of physical education, vol. 43, no. 3, pp. 39-45, 2019.

[3] Y. Kim and A. Bruland, "Analysis and evaluation of tunnel contour quality index," Automation in Construction, vol. 99, pp. 223-237, 2019.

[4] Q. Yan and Q. Wang, "Construction of smart city development level measurement index system," Statistics and decision making, vol. 34, no. 11, pp. 33-36, 2018.

[5] Z. Ghaderi, M. Rajabi, and M. Walker, "Psychic income benefits of small-scale sports events: host community perspectives," European Sport Management Quarterly, vol. 3, pp. 1-21, 2021.

[6] L. Jian, "Analysis on the development of sports event communication under the new media environment," Sports fashion, vol. 396, no. 12, pp. 218-219, 2020.

[7] M. Yang, H. Junya, and R. Luo, "Evolution path and trend analysis of foreign sports event tourism research," Journal of Xi'an Institute of physical education, vol. 37, no. 1, pp. 78-85, 2020.

[8] Q. Tan, B. Jingshan, and C. Chaoyu, "Calibration method of design response spectrum based on simulated annealing algorithm," Earthquake Engineering and Engineering Vibration, vol. 40, no. 1, pp. 158-164, 2020.

[9] Z. Youhao and Y. Zheng, "Multisensor information fusion technology based on projection pursuit simulated annealing," Sensor technology and application, vol. 8, no. 2, p. 12, 2020.

[10] B. Pueo, J. J. Lopez, and J. M. Jimenez-Olmedo, “Audio-based system for automatic measurement of jump height in sports science," Sensors, vol. 19, no. 11, p. 2543, 2019.

[11] B. A. Ahmed, "The relation of the motive sport achievement and self-confidence with the level of achievement of throwing events in athletics," International Journal of Psychosocial Rehabilitation, vol. 24, no. 4, pp. 6919-6931, 2020.

[12] T. Eski, "Examining winter sports awareness level of university students receiving sports education," Cypriot Journal of Educational Sciences, vol. 14, no. 4, pp. 630-640, 2019.

[13] M. Moital, A. Bain, and H. Thomas, "Summary of cognitive, affective, and behavioural outcomes of consuming prestigious 
sports events," Sport Management Review, vol. 22, no. 5, pp. 652-666, 2019.

[14] K. H. Lee, J. S. Lee, B. C. Lee, and E. H. Cho, "Relative weights of physical strength factors in sports events: focused on similarity sports events group according to the sports physiological view," Applied Sciences, vol. 10, no. 24, p. 9131, 2020.

[15] M. Gunduz and H. Laitinen, "Observation based safety performance indexing method for construction industry - validation with SMEs," KSCE Journal of Civil Engineering, vol. 22, no. 2, pp. 440-446, 2018.

[16] R. Socha and B. Winiewski, "Safety of mass sports events," ASEJ Scientific Journal of Bielsko-Biala School of Finance and Law, vol. 23, no. 1, pp. 42-44, 2019.

[17] M. Wunderlich, "Innovative approaches in sports sciencelexicon-based sentiment analysis as a tool to analyze sportsrelated twitter communication," Applied Sciences, vol. 10, no. 2, p. 431, 2020.

[18] W. H. Finch and B. F. French, "A simulation investigation of the performance of invariance assessment using equivalence testing procedures," Structural Equation Modeling, vol. 25, no. 5-6, pp. 673-686, 2018.

[19] A. V. Iglin, "International legal basis of using sports for development and peace," Moscow Journal of International Law, vol. 2, pp. 102-109, 2020.

[20] A. Yefanov, "Politicization of sports through media: syncretism of social institutions," Theoretical and Practical Issues of Journalism, vol. 10, no. 1, pp. 130-140, 2021.

[21] C. O. Sereati, A. Sumari, T. Adiono, and A. S. Ahmad, "Architecture design for a multi-sensor information fusion processor," TELKOMNIKA Indonesian Journal of Electrical Engineering, vol. 17, no. 1, pp. 362-369, 2019.

[22] L. Demyanova, I. Usova, A. Tashchiyan, N. Ryzhkin, and S. Demyanov, "Role of sports tourism in the school children health improvement," InE3S Web of Conferences, vol. 273, p. 09037, 2021.

[23] R. Nagovitsyn, A. Osipov, M. Kudryavtsev, O. Antamoshkin, and L. Glinchikova, "The increase of physical activity in persons using sports grounds for physical training," Human Sport Medicine, vol. 20, no. 1, pp. 100-105, 2020.

[24] C. O. Jang and B. J. Kim, "Relationship between non-verbal communication of middle school sports club instructors and the commitment and impact on class satisfaction," Journal of Korean Society for the Study of Physical Education, vol. 25, no. 3, pp. 257-271, 2020.

[25] R. Wilson and C. B. Mayhorn, "On the field: examining differences in video format in sports media viewing," Proceedings of the Human Factors and Ergonomics Society Annual Meeting, vol. 64, no. 1, pp. 781-785, 2020.

[26] M. Karayol and M. Taze, "Investigation of entrepreneurship levels and employability perception of undergraduate students studying sports sciences," International Education Studies, vol. 13, no. 5, pp. 35-36, 2020.

[27] B. Liang, L. Wei, and L. Junfeng, "Study on the construction of ecological risk assessment system for urban hosting large-scale sports events," Journal of Chengdu Institute of physical education, vol. 46, no. 2, pp. 34-41, 2020.
[28] D. Yi and D. Jian, "Index dimension analysis and enlightenment of building London into a world-famous Sports City," Journal of Shanghai Institute of physical education, vol. 43, no. 1, pp. 65-71, 2019.

[29] Y. Huili, "Research on coupling time series evolution and influencing factors of sports events and host city development," China Sports Science and technology, vol. 55, no. 3, pp. 53-60, 2019. 